\title{
Editorial \\ Augmenting Epithelial Barrier Function
}

James M. Mullin,*\#

\begin{abstract}
The Lankenau Institute for Medical Research and The Division of Gastroenterology, Lankenau Hospital, Wynnewood, PA 19096 USA
\end{abstract}

The simplest multicellular animal organisms, coelenterates, are essentially floating intestines in the sea. More fundamentally, they are 'barrier tubes' that attempt to compartmentalize the environment they exist in. It is said that ontogeny recapitulates phylogeny. It is thus interesting that the blastocyst, among the earliest multicellular embryonic structures, is essentially a sac comprised of polarizing cells. Like the coelenterate, it is also compartmentalizing the environment that it exists in, creating an internal fluid compartment distinct from an external one. So on both an evolutionary basis and a developmental basis, the singular importance of fluid compartmentation and barrier cell linings was a concept that nature apparently grabbed hold of with both hands and never let go, as it kept ramifying the theme. The human body is in a sense a manifold of many different sacs and tubes: a respiratory tract, a GI tract, a urinary tract, the skin, a reproductive tract, a vasculature, a central nervous system encased in a medium (cerebrospinal fluid) quite distinct from blood. We are intimately and fundamentally divided into luminal and abluminal compartments.

A short word about the barriers separating the compartments. They can be multilayered (the skin) or single-cellthick (the small intestine). They can be highly 'porous' (a capillary vessel or the ileum) or extremely 'tight' (the urinary bladder or esophagus). But despite all their various formats, they ultimately consist of two components - the cells per se and the junctional seals that surround them in gasket-like fashion. For whatever the barrier, there are two ways for any solute to pass across - through the cells or through the junctional seals. A quiet revolution in biomedical thinking over the past 60 years was the dawning realization that tight junctions (TJs) exist as a barrier element, that they are composed of distinct proteins (occludin, claudins, etc), and the fact that they are not static but subject to myriad pathways of intracellular signaling and regulation (excellently reviewed in Schneeberger and Lynch, 2004) [1]. Pathogens had apparently figured this out long before we did for many have learned to target the TJs as preferred entry points to get across tissue barriers [2, 3]. They not only frequently like to latch onto the barrier at these sites, but from viruses to bacteria to even (eukaryotic) dust mites, they have

*Address correspondence to this author at the The Lankenau Institute for Medical Research and The Division of Gastroenterology, Lankenau Hospital, Wynnewood, PA 19096 USA; Tel: 484-476-2703; Fax: 484-476-2205; E-mail: MullinJ@mlhs.org

"Guest editor derived ingenious ways to get our junctional seals to 'open up' (or fall apart) and then come on through into the stroma and vasculature, much to our chagrin [4-6].

A common theme of many of our luminal compartments is communication with the external environment, a relatively rough-and-tumble place with many unsavory elements. In fact, our luminal compartments are typically replete with a "rogues' gallery" of microorganisms and antigens, in contrast with the relatively pristine, microorganism-free abluminal compartment - the interstitium and bloodstream. The rogues-gallery of pathogens sitting in our luminal compartments has moreover through the millennia become audaciously adept at getting across our mucosal linings [7]. From their point of view, they have a dodgy existence in a mercurial luminal environment compared to what they may perceive as the climate-controlled, nutritional "Promised Land" on the other side of the mucosa. If luminal compartments are typified by a robust microbial/antigen population, the abluminal compartment is interestingly the home of the immune system, an entity that is typically not microbe- or antigenfriendly. This intriguing contra-position across epithelial barriers is the medical border 'flash point' behind much of the morbidity (and mortality) that afflicts us. The phrase "good fences make good neighbors" never held more validity than it does for our epithelial tissues, and the two very different worlds that they separate. The article that Slattery et al. contribute here on immunosuppressant drugs enhancing barrier function certainly emphasizes the good fence/good neighbors directive in our own bodies.

The opening article by Farrell et al. in this special issue highlights the medical importance of this fundamental compartmentation / barrier reality. They focus on the GI tract as an example of the surprising array of diseases and syndromes that can arise directly from barrier compromise. They then 'expand out' - in an epithelial tissue framework to consider multi-organ failure (a most prominent, immediate cause of death for the hospital in-patient), as itself a text book case of barrier failure on a grand scale. Their contribution lays a very nice groundwork for why devising means for improvement of epithelial barriers - the theme of this special issue - is so important in disease prevention and therapy.

Although we have so far considered microbes and immune response, don't forget the physiology. More basic than the infectious disease/immunology considerations, is simply 
the 'prime directive' for most epithelial tissues - move solutes and fluid from one side of the barrier to the other, in other words, create gradients. It is this thermodynamic work at the expense of ATP that makes our various tissue compartments the unique places that they are. The epithelial layers engage in this thermodynamic work every minute of the day, every day of the year, every year of our lives. The bioenergetic heat from these processes has a good deal to do with us being warm-blooded. So consider too that any subtle sundering of barriers has a basic thermodynamic cost to the organism totally aside from the immunologic downside. It's simply not good to pump things from A to B and then watch them back-leak from B to A. It's tough on gradients. It's not good for homeostasis and bioenergetics. It generally makes for a bad day.

So pathogens and even non-infectious disease processes (cancer and inflammation, for example) have myriad ways of making our epithelial barriers leaky [4]. In fact over the last 25 years, research on junctional barriers has unearthed an impressive array of extracellular ligands such as the proinflammatory cytokines and intracellular signaling pathways in the epithelial cell (protein kinase $\mathrm{C}$, myosin light chain kinase, Raf/MEK/Erk, etc.) that are all too good at making our linings leaky $[4,8-10]$. This is in marked contrast to the relative paucity of means that have so far been found to make our junctional seals and our linings tighter - the central theme of this special journal issue [11]. Some agents, e.g. Epidermal Growth Factor and glucorticoids, [12-16] are intriguing agents for inducing barrier tightening in their own right but don't fill-the-bill when it comes to useful medical interventions - simply too much downside and baggage. Other approaches that are described and discussed herein zinc (see the article by Zhou and Zhong), probiotics (see the article by Madsen), indole (see the article by Bansal et al.), glutamine (see the article by Rao and Samak), quercetin (see the article by Chuenkitiyanon et al.) and methioninerestriction (see the article by Wang and Mullin) - are interestingly all nutritional in nature. While this may not necessarily be good for intellectual property rights, it could be quite a boon for the average individual who needs inexpensive and readily-administered therapeutic interventions. Each one of these agents has its own anecdotal (or better) 'resume' attesting to beneficial health effects. Stroll into any health food / herbal remedy store and you'll find these very agents featured. Zinc (think Cold Eeze lozenges) is already widely used as a prophyllactic against upper respiratory infections. Probiotics (heard of Activia lately?) have been used extensively as treatments for various GI upsets. Flavonoids and polyphenols (ever drink red wine or green tea?) are absolute mainstays of the off-label pharmacopeia, ascribed for a wide range of diseases. Glutamine has found recent enteral use, even within hospitals, for general wound repair. Methioninerestriction (think 'vegetarian' here) has been found to extend lifespan and forestall age-related disease. The jury is certainly still 'out' on whether these various remedies can in fact truly 'deliver' clinically, but if they can do so even partially, their potential for global health impact is significant indeed. Their very diversity, in terms of chemical structure and their source in nature, implies that they target something fundamental in us, and therefore their having a salutary effect on entities as fundamental as epithelial and endothelial barriers isn't all that surprising.
Agents such as sirolimus or Hypoxia-Inducible-Factor (see the article by Kelly and Colgan, and by Slattery et al.) are not in any health food store (in my own neighborhood) and are certainly not in a "nutritional" category but highlight something else all-so-interesting about compounds that augment barrier function - they frequently act as entrenched, built-in countermeasures to agents that induce leakiness. HIF, acting as it does in situations of anoxia - a frequent 'bed-fellow' of ischemia - is one known means of countering the avalanche of proinflammatory cytokines that we now know full well can assault epithelial and endothelial barrier integrity in these conditions.

I realize that the study of these agents can appear to some as mere 'phenomenology' (a charge that certainly appears in more than a few grant proposal reviews I've received over the years) but consider this - the tight junction can be composed of as many as 26 different claudins, and that excludes the potential role of occludin (let alone tricellulin, cingulin, 7H6, ZO-1, etc.). If one was to approach the field of barrier enhancement using the fashionable and favored molecular biological "bottom-up" 'play-book', exactly how does one deal with this complexity? If, as seems likely, claudins work in homo- and heterodimer, zipper-like interplay, you have a permutation situation mathematically. So if one is planning on designing a better barrier by transgenic overexpression or knockout, how exactly does one deal with 26 (or more) factorial? The tight junction is the true modern day "Gordian Knot" and researchers (or reviewers) should not spurn nutritional or herbal remedies when they are serving as invaluable guideposts to what IS a better barrier (in ALL its ramifications). Moreover, while molecular physiologists are still working on tight junctional complexity two generations from now, it might be nice to know that one has done something beneficially substantive and real for people in one's own lifetime here today.

\section{REFERENCES}

[1] Schneeberger EE, Lynch RD. The tight junction: a multifunctional complex. Am J Physiol Cell Physiol 2004; 286: C1213-28.

[2] Guttman JA, Finlay BB. Tight junctions as targets of infectious agents. Biochim Biophys Acta 2009; 4: 832-41.

[3] Gonzalez-Mariscal L, Garay E, Lechuga S. Virus interaction with the apical junctional complex. Front Biosci 2009; 14: 731-68.

[4] Mullin JM, Agostino N, Rendon-Huerta E, Thornton JJ. Keynote review: epithelial and endothelial barriers in human disease. Drug Discov Today 2005; 10: 395-408.

[5] Sawada N, Murata M, Kikuchi K, et al. Tight junctions and human diseases. Med Electron Microsc 2003; 36: 147-56.

[6] Wan H, Winton HL, Soeller C, et al. Der p 1 facilitates transepithelial allergen delivery by disruption of tight junctions. J Clin Invest 1999; 104: 123-33.

[7] Marchiando AM, Graham WV, Turner JR. Epithelial barriers in homeostasis and disease. Annu Rev Pathol 2010; 5: 119-44.

[8] Edelblum KL, Turner JR. The tight junction in inflammatory disease: communication breakdown. Curr Opin Pharmacol 2009; 9: 715-20.

[9] Samarin S, Nusrat A. Regulation of epithelial apical junctional complex by Rho family GTPases. Front Biosci 2009; 14: 1129-42.

[10] Steed E, Balda MS, Matter K. Dynamics and functions of tight junctions. Trends Cell Biol 2010; 20: 142-9.

[11] Hering NA, Schulzke JD. Therapeutic options to modulate barrier defects in inflammatory bowel disease. Dig Dis 2009; 27: 450-4.

[12] Felinski EA, Cox AE, Phillips BE, Antonetti DA. Glucocorticoids induce transactivation of tight junction genes occludin and claudin5 in retinal endothelial cells via a novel cis-element. Exp Eye Res 2008; 86: 867-78. 
[13] Sheth P, Seth A, Thangavel M, Basuroy S, Rao RK. Epidermal growth factor prevents acetaldehyde-induced paracellular permeability in Caco-2 cell monolayer. Alcohol Clin Exp Res 2004; 28: 797-804.

[14] Singer KL, Stevenson BR, Woo PL, Firestone GL. Relationship of serine/threonine phosphorylation/dephosphorylation signaling to glucocorticoid regulation of tight junction permeability and ZO-1 distribution in nontransformed mammary epithelial cells. J Biol Chem 1994; 269: 16108-15.
[15] Soler AP, Laughlin KV, Mullin JM. Effects of epidermal growth factor versus phorbol ester on kidney epithelial (LLC-PK1) tight junction permeability and cell division. Exp Cell Res 1993; 207: 398-406.

[16] Singh AB, Harris RC. Epidermal growth factor receptor activation differentially regulates claudinexpression and enhances transepithelial resistance in Madin-Darby canine kidneycells. J Biol Chem 2004 279: $3543-52$.

(C) James M. Mullin; Licensee Bentham Open.

This is an open access article licensed under the terms of the Creative Commons Attribution Non-Commercial License (http://creativecommons.org/licenses/ by-nc/3.0/) which permits unrestricted, non-commercial use, distribution and reproduction in any medium, provided the work is properly cited. 